\title{
A foldable electrode array for 3D recording of deep-seated abnormal brain cavities
}

\author{
Dries Kil ${ }^{1}$, Philippe De Vloo ${ }^{2}$, Guy Fierens ${ }^{3}$, Frederik Ceyssens', Borbála Hunyadi ${ }^{3}$, \\ Alexander Bertrand ${ }^{3}$, Bart Nuttin ${ }^{2}$ and Robert Puers ${ }^{1}$ \\ ${ }^{1}$ Department of ESAT-MICAS, KU Leuven, Leuven, Belgium \\ ${ }^{2}$ Laboratory of Experimental Neurosurgery and Neuroanatomy, KU Leuven, Leuven, Belgium \\ ${ }^{3}$ Department of ESAT-STADIUS, KU Leuven, Leuven, Belgium \\ E-mail: dries.kil@esat.kuleuven.be
}

\begin{abstract}
Objective. This study describes the design and microfabrication of a foldable thin-film neural implant and investigates its suitability for electrical recording of deep-lying brain cavity walls. Approach. A new type of foldable neural electrode array is presented, which can be inserted through a cannula. The microfabricated electrode is specifically designed for electrical recording of the cavity wall of thalamic lesions resulting from stroke. The proof-of-concept is demonstrated by measurements in rat brain cavities. On implantation, the electrode array unfolds in the brain cavity, contacting the cavity walls and allowing recording at different anatomical locations. A three-layer microfabrication process based on UV-lithography and Reactive Ion Etching is described. Electrochemical characterization of the electrode is performed in addition to an in vivo experiment in which the implantation procedure and the unfolding of the electrode are tested and visualized.

Main results. Electrochemical characterization validated the suitability of the electrode for in vivo use. CT imaging confirmed the unfolding of the electrode in the brain cavity and analysis of recorded local field potentials showed the ability to record neural signals of biological origin.

Significance. The conducted research confirms that it is possible to record neural activity from the inside wall of brain cavities at various anatomical locations after a single implantation procedure. This opens up possibilities towards research of abnormal brain cavities and the clinical conditions associated with them, such as central post-stroke pain.
\end{abstract}

Keywords: Neural implant, polyimide, microfabrication, foldable, unfoldable, brain cavity, central post-stroke pain, local field potential 


\section{Introduction}

For the better part of last decade neural implant research has been booming. A plethora of novel high resolution, highly biocompatible neural electronic interfaces are being designed with applications spanning a vast range of scientific fields. These include, among others, basic research concerning the fundamental functionality of the healthy nervous system [1], treatment of neurological conditions (depression, Parkinson's disease,...) [2] and more recently the development of neuroprosthetics, which are devices that can substitute a motor, sensory or cognitive modality which is damaged as a result of trauma or neurodegenerative disorders. [3]

Naturally, due to the rapid evolution of the related scientific fields, the complexity of the applications increases, which in turn gives rise to the need for more sophisticated electrode designs. A very interesting application which requires the development of such a custom neural implant is the electrical mapping and stimulation of abnormal brain cavity (aBC) walls. aBC's typically develop after neural loss caused by e.g. trauma, surgery, stroke or infections, and can be accompanied by pathological activity in the affected neuronal circuits, resulting in a series of possible symptoms such as chronic pain or impaired motor function [4]. These effects are typically treated by medication [5] or neuromodulation [6], but these techniques are only effective in a limited number of cases. There is limited evidence that, at least in some cases, clinical symptoms could be alleviated by electrical stimulation of the cavity wall. [7]

Electrical stimulation of an infarcted motor cortex in a rat stroke model significantly reduces motor deficits [7,8]. In a similar model, neural signals were recorded from the perilesional cortex and modulated volitionally to learn neuroprosthetics control, with a learning rate and a task-related modulation comparable to the intact cortex more distant from the aBC [9]. Furthermore, deep brain stimulation (DBS) in the close vicinity of the aBC has been used successfully as a complementary therapy in patients with incomplete effect of therapeutic electrolytic lesions [10,11] and radiosurgery [12].

In this paper we describe the design, fabrication, electrical characterization and in vivo validation of a novel foldable micro-electrode which is tailor-made for implantation into subcortical cavities. The goal is to develop a highly flexible and foldable electrode array which forms an intimate electrical contact with the brain cavity wall at various anatomical locations and can be inserted through a cannula. Other examples of deployable multi-contact electrodes have been developed by $C$. Lieber et al [13]. In order to obtain a similar result with conventional rodent micro-electrodes in this application, multiple electrodes need to be implanted which could cause additional mechanical trauma to healthy neural tissue. It is also very challenging to position the electrodes exactly on the $\mathrm{aBC}$ wall. As the presented electrode is designed as a flat structure a bespoke packaging procedure was developed in which the electrode is temporarily folded onto itself. This strongly reduces its footprint for insertion and ensures minimal damage to the healthy tissue surrounding the implantation trajectory. The general strategy involves temporary folding up the flexible electrode and inserting it in a cannula, after which the cannula is stereotaxically inserted into the target cavity. Subsequently, the electrode is pushed through the cannula and into the cavity using a stylet, where it unfolds and takes on a three-dimensional shape conform to the cavity wall. 
The multi-contact implant then allows electrical stimulation as well as recording of the cavity wall at various anatomical locations after a single implantation procedure.

An extended overview on the electrode design and fabrication process as well as on the implantation procedure and electrical characterization is provided. Lastly an in vivo experiment was performed in which the electrode was implanted using the novel procedure. Correct unfolding of the electrode was validated by means of CT imaging. Local Field Potentials (LFP) were recorded to validate the correct operation of the electrode after implantation.

\section{Methods}

\subsection{Neural implant design}

The implant used in this application was designed for recording of neural activity in deep-seated brain aBCs in rats. With the eventual goal of reducing the symptoms associated with the presence of aBCs two requirements are set which the electrode design has to fulfill. A first requirement is the formation of physical contact between the implant and the aBC wall after implantation. The implant should contain multiple electrode contacts that cover a significant part of the aBC. Secondly, all of this should be achieved after a single implantation procedure, omitting the use of multiple micro-electrodes as described before.

Due to the irregular shape of brain cavities we opted for the development of a highly flexible and expandable implant. This not only permits to fold the electrode to a smaller size and compress it for implantation, but it also allows to design it in such a way that after implantation it unfolds again and regains a three-dimensional shape conform to the brain cavity wall.

Keeping these guidelines in mind the neural implant was designed as a flower-like structure consisting of 7 extended leaves, each containing one platinum electrode contact, which are joined in a central hinging point. This allows the implant to take on a hemi-spherical shape without inducing wrinkles or excessive stress, which is not possible with a conventional planar thin film design [14]. Extra bridging structures around the outer edge of the leaves add extra strength and rigidity and assist in the unfolding of the implant after it is lodged in the cavity.

The flower shaped implant-tip (fig. 1) has a diameter of $3 \mathrm{~mm}$ which is optimized with respect to the size of the target cavity, as present in the animals used for the in vivo experiment. The conductors are routed to a 9-channel Omnetics connector (Omnetics Connector Corporation, USA) through a lead with a length of $40 \mathrm{~mm}$ which allows easy fixation of the connector assembly on the back of the skull. The distance between the implantation site and the final location of the connector was maximized in order to minimize the artefacts it generates during X-ray imaging. The whole device is fabricated using a MEMS-compatible fabrication process, allowing high volume production with a high turnover rate.

Figure 1 shows a finished implant resulting from the fabrication process. After the implant was released from the silicon carrier wafer, an Omnetics connector was soldered onto the bond pads. A detailed microfabrication process is described in section 2.3. 


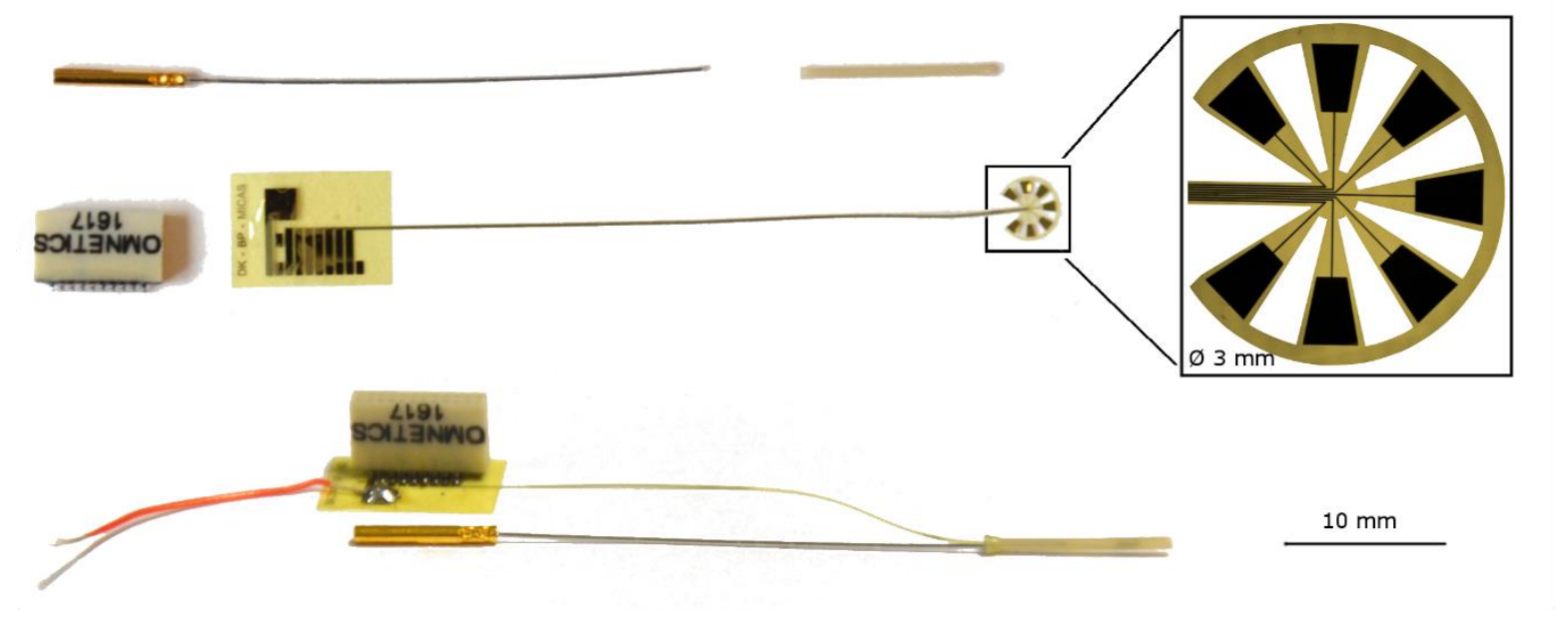

Fig. 1. Overview of the device before and after assembly. The insert shows a close-up of the electrode tip

\subsection{Material choice}

Polyimide (PI) (HD MicroSystems ${ }^{\mathrm{TM}}$ PI-2611) was chosen as the base material for the neural implant. Its dielectric properties, high strength under bending and biocompatibility make it an excellent candidate for this application. As described by B. Rubehn et al., PI is stable in a physiological environment and is non-toxic to the surrounding cells [15]. The flexibility of PI significantly increases the mechanical compliance between the device and the soft biological tissue surrounding it, which in turn prevents additional micromotions which often lead to inflammation and gliosis and eventually encapsulate the device in electrically isolating scar tissue.

Polyimide was chosen over other alternatives such as Parylene $\mathrm{C}$ due to its superior mechanical properties [16]. The tensile strength of PI-2611 (390 MPa) is almost a factor 6 higher than Parylene C's. Furthermore its Young's modulus is also about 2.6 times higher. This allows small bending radii to be imposed to the polyimide without inducing any cracks [17]. The thermal expansion coefficient of this PI ( $3 \mathrm{ppm} / \mathrm{K})$ is also comparable to that of silicon, which prevents bending of the devices after releasing them from the substrate.

Platinum was chosen as the material of choice for the conductors due to its biocompatibility, inertness in biological environments and high charge injection limit [18]. 


\subsection{Microfabrication process}

The neural implants were fabricated using an established two-mask, three-layer microfabrication process on silicon carrier wafers [19]. The following workflow is applied:

- Standard 4 inch silicon wafers are thoroughly cleaned using piranha etchant $\left(4 \mathrm{H}_{2} \mathrm{SO}_{4}: 1\right.$ $\mathrm{H}_{2} \mathrm{O}_{2}$ ) to remove organic contaminants. Afterwards a HF-dip (2\% HF) is performed followed by a rinse in DI water. The substrate is dried using purified nitrogen.

- A $400 \mathrm{~nm}$ thin layer of silicon oxide is grown (wet thermal oxidation) with the purpose of serving as a sacrificial layer. In a later step the oxide layer is chemically removed in HF, releasing the devices from the silicon carrier wafer.

- The first insulation layer is deposited by spincoating polyimide (HD microsystems PI2611) at $2800 \mathrm{rpm}$ on top of the silicon oxide layer, yielding a $7 \mu \mathrm{m}$ thick layer. The first layer of PI is cured at $205^{\circ} \mathrm{C}$ to increase the reactivity to the second PI layer which is deposited in a later step. This improves the lifetime of the device with a factor of 7.5 as was proven by F. Ceyssens et al. [20]

- The $400 \mathrm{~nm}$ thick Pt conductors are deposited by sputter coating on a lithographically patterned photoresist bilayer (LOR10B / S1818). The lift-off was completed by soaking the wafers in NMP (n-methyl-2-pyrrolidone) overnight at room temperature.

- The second layer of PI is deposited and fully cured at $340{ }^{\circ} \mathrm{C}$

- The device shape is defined by reactive ion etching (RIE) of the parylene $\mathrm{C}$ using an aluminum hard mask. The device outline was lithographically patterned using a negative photoresist on top of which a $40 \mathrm{~nm}$ thick layer of aluminum was thermally evaporated. After a quick lift-off step the wafers were ready for RIE. As platinum is not etched by the RIE plasma, it also functions as an etch stop, which opens up the electrode contacts and bond pads.

- In a final step the wafer is soaked in a $1 \%$ HF solution which removes the Al hard mask and underetches the sacrificial silicon oxide layer. After 1 hour of etching the adhesion between the PI electrode and the carrier wafer is reduced and the electrodes can be peeled off the carrier wafer using tweezers.

- After release a 9 channel Omnetics connector is soldered directly onto the bond pads. The whole connector assembly is then isolated using a biocompatible epoxy-based underfill (Epo-Tek 302-3M).

After completing the fabrication process the implants are stored in $70 \%$ ethanol for disinfection purposes. 


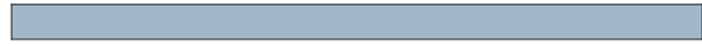

(1) 4" silicon wafer substrate

(2) Sacrificial $\mathrm{SiO}_{2}$ deposition

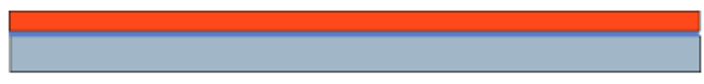

(3) PI-2611 spin-coating

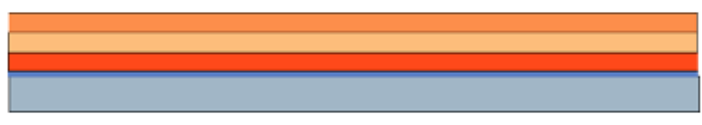

(4) Photoresist spin-coating (LOR10B / S1818)

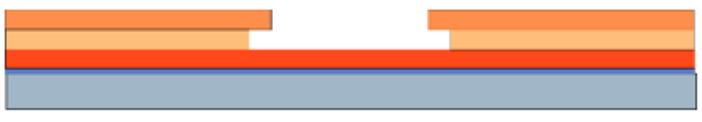

(5) Photoresist exposure and development

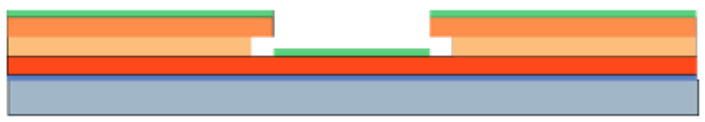

(6) Platinum sputter deposition

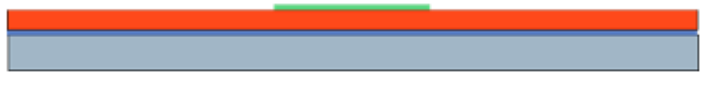

(7) Lift-off

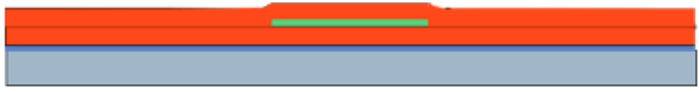

(8) PI-2611 spin-coating

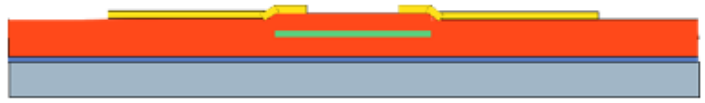

(9) Metal mask deposition

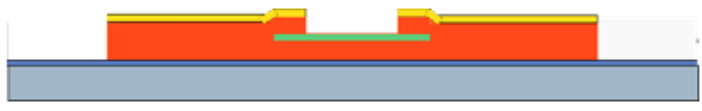

(10) Reactive ion etching of PI

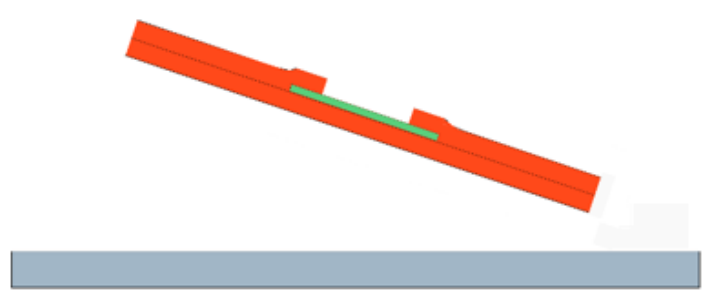

(11) Oxide strip

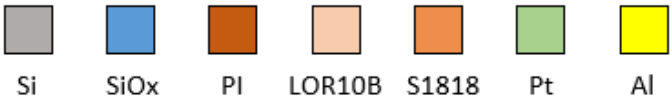

Fig. 2. Microfabrication process

\subsection{Electrochemical characterization}

The electrochemical behavior of the neural implant was analyzed using cyclic voltammetry and electrochemical impedance spectroscopy (EIS). All measurements were performed using a VersaSTAT 4 (Princeton Applied Research) potentiostat. A 3 electrode configuration was used consisting of a working, counter and reference electrode in phosphate buffered saline (PBS). A platinum coil and $\mathrm{Ag} / \mathrm{AgCl}$ electrode were used as a counter and reference electrode, respectively.

The concept of using electrochemistry to validate the operating window of neural electrodes was first introduced by Barry Brummer in the 1970s. He stated that platinum electrodes could be safely used in the so called 'water window', which is defined as the potential range limited by water reduction (hydrogen evolution) and water oxidation (oxygen evolution). Operating the electrode within these limits assumes that any electrochemical reactions that occur (i.e. platinum oxidation,...) are reversible [21-24]. This electrochemical window was determined using cyclic voltammetry. The voltammogram was recorded in the 'water window' at a scan rate of 100 $\mathrm{mV} / \mathrm{sec}$. 
The impedance of all electrode contacts was measured in a frequency range from $0.1 \mathrm{~Hz}$ to 100 $\mathrm{kHz}$ with a sinusoidal input voltage of $100 \mathrm{mV}_{\mathrm{pp}}$. To analyze the EIS data, an equivalent circuit model was fitted to the data, providing further insight into the charge transfer properties of the electrode. A Randels' circuit was chosen as the optimal model and is composed of a solution resistance $\left(R_{s}\right)$ in series with a charge transfer resistance $\left(R_{c t}\right)$ which is in parallel with a double layer capacitor $\left(\mathrm{C}_{\mathrm{dl}}\right)$.

\subsection{Packaging and controlled unfolding}

Prior to implantation, the electrode is inserted into a cannula using a thin titanium stylet. Using the stylet as a piston, force is applied to the center joint of the flower-like structure (fig. 1) as it is positioned above the cannula. The electrode then folds onto itself and around the stylet as it is pushed through the cannula. Due to the symmetric design of the electrode, the trapezoidal shape of the extensions and the presence of the bridging structures, the electrode tip folds in a controlled manner as depicted in figure 3 . The extensions of the electrode tip overlap nicely and the bridging structures tend to fold up in between. As the electrode is advanced through the cannula, these bridging structures exert a spring-like behavior and force the electrode to unfold back to a shape which is limited by the space available in the cavity.

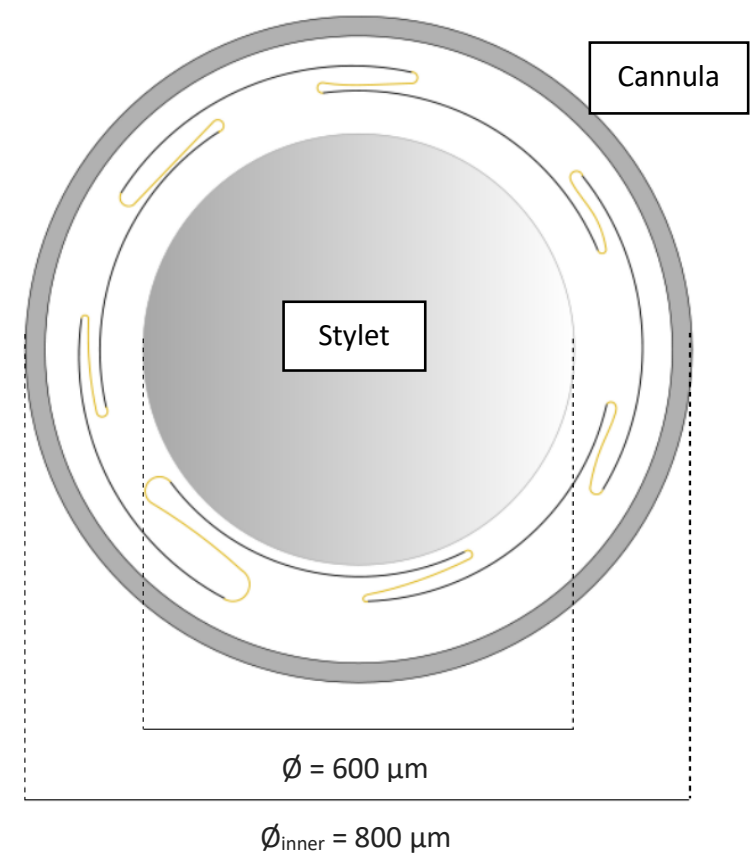

Fig. 3. Graphical representation of the electrode folded into the cannula (cross section). The bridging structures are depicted in yellow. 


\subsection{Implantation procedure}

A graphical representation of the implantation procedure is depicted in figure 4. Prior to the implantation the electrode is inserted into the cannula as described in section 2.5 (a). The assembled device (fig.1) is then mounted to the stereotaxic frame (b). Pre-operative CT (Computed Tomography) and MR (Magnetic Resonance) imaging are used to calculate the target coordinates. Once in the right position (c), the dura is punctured and the cannula is advanced into the brain until the aBC is reached (d). Afterwards, a second stereotaxic arm is positioned above the stylet and used to advance the stylet through the cannula (e). As the electrode is pushed beyond the distal cannula opening, the thin PI bridging structures that connect the electrode-contacts force the electrode to unfold, thereby making contact with the aBC wall (f). After the implantation, the second stereotaxic arm is removed (g), the cannula is fixed to the skull using dental cement (h) and the stylet is retracted (i) and the connector and cannula are fixed onto the skull using dental cement. By example the implanted device is depicted in figure 5.

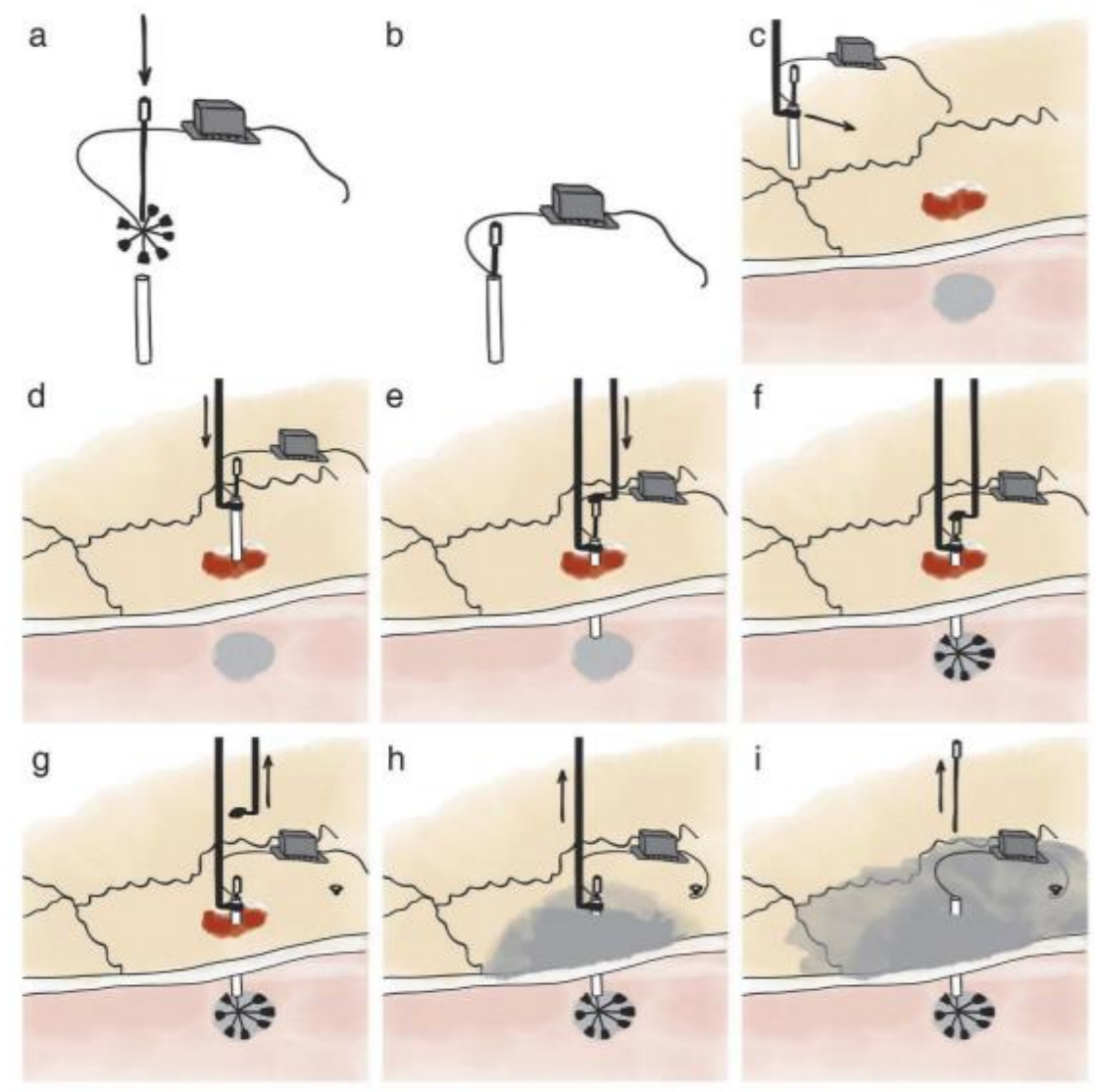

Figure 4. Graphical representation of the implantation procedure. 


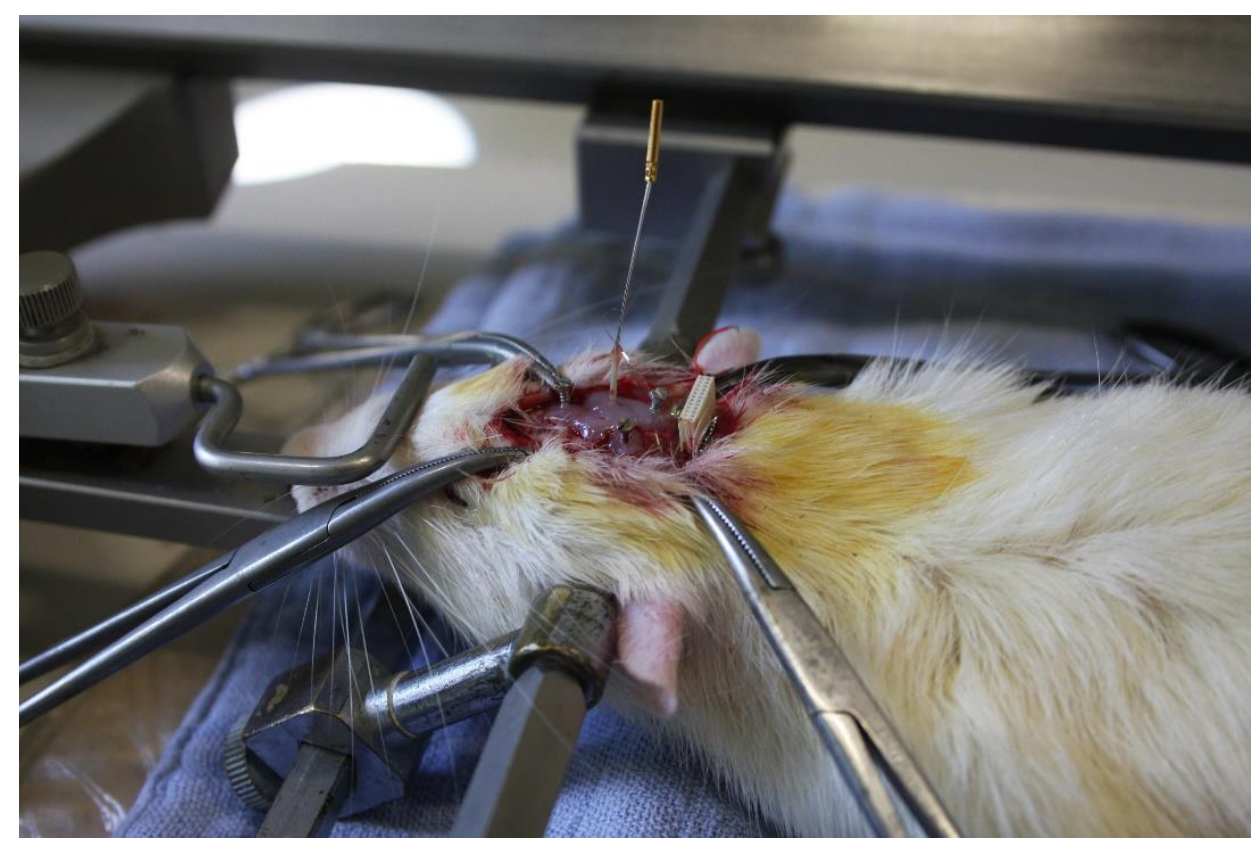

Fig. 5. Image of the implanted device showing the bone screws, cannula, stylet and Omnetics connector

\subsection{In vivo experiment}

In order to assess the correct operation of the electrode assembly an in vivo experiment was set up. The goal was twofold, on one hand the implantation procedure and the correct unfolding of the electrode is validated using imaging techniques, on the other hand LFPs are recorded and analyzed to check if signals of biological origin can be recorded.

The experiment was performed on male Sprague-Dawley rats $(\mathrm{N}=24)$. They were housed in pairs, separated by a cage divider, in a controlled room with a constant temperature of $20{ }^{\circ} \mathrm{C}$ and a $14: 10$ light:dark cycle. Food was restricted to $16 \mathrm{~g}$ /day to keep the animals thin enough to fit the in vivo small animal MR and CT scanner, while water was distributed ad libitum. The research project was in accordance with the Belgian and European laws, guidelines and policies for animal experimentation, housing and care (Belgian Royal Decree of 29 May and European Directive 2010/63/EU on the protection of animals used for scientific purposes of 20 October 2010).

Four weeks after inducing an electrolytic lesion in the right sensory thalamus (Table 1), CT and MRI scans were acquired and processed to determine the size and location of the created aBC. Five weeks after lesion induction the described electrode was implanted in the animals in which a fluid-filled aBC with a dorsoventral dimension of at least $1.5 \mathrm{~mm}(\mathrm{~N}=21)$ was detected using individualized stereotaxic planning. One day and two weeks after the implantation surgery the position of the electrode was confirmed using a CT scan, which was co-registered to the preoperative MRI. 
Table 1. Electrolysis coordinates (in $\mathrm{mm}$, relative to bregma)

\begin{tabular}{l|l|l|l|l}
\hline $\begin{array}{l}\text { Order of electrode } \\
\text { insertion }\end{array}$ & $\begin{array}{l}\text { Anteroposterior } \\
\text { coordinates }\end{array}$ & $\begin{array}{l}\text { Mediolateral } \\
\text { coordinates }\end{array}$ & $\begin{array}{l}\text { Dorsoventral } \\
\text { coordinates }\end{array}$ & Anatomical region \\
\hline 1 & -2.40 & +2.00 & 6.25 & $\begin{array}{l}\text { Ventrolateral thalamic } \\
\text { nucleus }\end{array}$ \\
\hline 2 & -3.20 & +2.00 & 6.50 & $\begin{array}{l}\text { Posterior thalamic } \\
\text { nucleus }\end{array}$ \\
\hline 3 & -4.00 & +2.00 & 6.25 & $\begin{array}{l}\text { Posterior thalamic } \\
\text { nucleus }\end{array}$ \\
\hline 4 & -2.40 & +3.50 & 6.25 & $\begin{array}{l}\text { Ventral posterolateral } \\
\text { thalamic nucleus }\end{array}$ \\
\hline 5 & -3.20 & +3.50 & 6.50 & $\begin{array}{l}\text { Ventral posterolateral } \\
\text { thalamic nucleus }\end{array}$ \\
\hline 6 & -4.00 & +3.50 & 6.25 & $\begin{array}{l}\text { Ventral posterolateral } \\
\text { thalamic nucleus }\end{array}$ \\
\hline
\end{tabular}

MR imaging was performed on a 9.4T small animal MR scanner (Biospec 94/20 USR Bruker, Ettlingen, Germany). T2 RARE images ( $\mathrm{TE}=14.1468 \mathrm{~ms}$ and $\mathrm{Tr}=1500 \mathrm{~ms}$ ) were taken with a isotropic spatial resolution of $125 \mu \mathrm{m}$.

In the weeks following the electrode implantation the animals were subjected to an open field experiment in which LFPs were recorded. Simultaneously a video was recorded which is used to segment the LFP signal into epochs in which the animal was moving or sitting still.

\subsection{In vivo experiment}

In order to assess the ability of the electrode to record signals of biological origin the animal was subjected to an open field experiment in which it could roam around in a $1 \mathrm{~m}^{2}$ wooden box without performing any tasks. By comparing the recorded LFPs during movement and resting of the rat it is possible to determine changes in the LFP spectrum. As the thalamus is involved in motor coordination, a significant change in the LFP spectrum indicates the biological nature of the recorded signals.

LFP were recorded using a wireless system consisting of a W16 wireless headstage receiver and data acquisition card in combination with MCRack software (Multi Channel Systems, Reutlingen, Germany). LFPs were recorded at a sampling frequency of $10 \mathrm{kHz}$ between each electrode contact and the reference electrode connected to the posterolateral anchor screw in the skull (fig. 4). The measured signals were amplified in the range of $1 \mathrm{~Hz}$ to $5 \mathrm{kHz}$ and digitized in the head stage before wireless transmission to the receiver. LFPs were acquired for 5 minutes during which a video was recorded at a framerate of 30 FPS (Sony HDR-AS15).

Prior to further analysis the raw data was pre-processed. The LFP signals are re-referenced in function of the common average to minimize the impact of the reference electrode activity. Afterwards, the data is notch filtered (at $50 \mathrm{~Hz}$ with a $-3 \mathrm{~dB}$ stopband bandwidth of $5 \mathrm{~Hz}$ ) and bandpass filtered (Butterworth filter of order 20 with $-3 \mathrm{~dB}$ cut-off at $0.5 \mathrm{~Hz}$ and $50 \mathrm{~Hz}$ ) to remove power-line interference and low- and high-frequency artefacts. Lastly, the recorded video is synchronized with the LFP data using a combined light emitting diode (LED) and transistor- 
transistor logic signal. After synchronization a custom video algorithm was used to quantify the animal's movement during the experiment. Using this algorithm the LFP signals are divided into timeframes corresponding to movement vs. resting.

After re-referencing, filtering and synchronization, outlier segments with strong signal peaks were removed from the data using the procedure in [25], and the resulting signal was divided in epochs of 1 second. Within each epoch, an instantaneous estimate of the power spectral density (PSD) in each electrode was computed based on a Hanning-weighted periodogram. Six different parameters were extracted from the average PSD (averaged ${ }^{1}$ across 7 electrodes) within each epoch: the rootmean-square (RMS) voltage (square root of the broadband power), and the relative power in 5 subbands (delta $(1-4 \mathrm{~Hz})$, theta $(4-8 \mathrm{~Hz})$, alpha $(8-15 \mathrm{~Hz})$, beta $(15-35 \mathrm{~Hz})$ and gamma1 $(35-50$ $\mathrm{Hz})$ ), relative to the total broadband power within the epoch.

\section{Results and discussion}

\subsection{Cyclic voltammetry}

In the case of the presented electrode, hydrogen and oxygen evolution respectively start at potentials of $-0.4 \mathrm{~V}$ and $1 \mathrm{~V}$ vs. $\mathrm{Ag} / \mathrm{AgCl}$, which is consistent with data gathered from literature $[26,27]$. The large step around $0.1 \mathrm{~V}$ vs. $\mathrm{Ag} / \mathrm{AgCl}$ can be attributed to the reduction of dissolved oxygen which is present in the solution [28]. Further, the voltammogram is featureless within the water window, meaning that no faradaic reactions occur and that current is delivered through the charging and discharging of the interfacial double layer. This capacitive charge injection mechanism proves that the system is suitable for cavity wall recordings. The charge transfer capacity of the Pt electrode, which is defined as the time-integral of the voltammogram between the limits of the water window, has a value in the order of $7.6 \pm 3.5 \mathrm{mC} / \mathrm{cm}^{2}$ which can be injected safely into the tissue. Both results confirm the operating range of the $\mathrm{Pt}$ electrodes under stimulation without producing irreversible faradaic reactions.

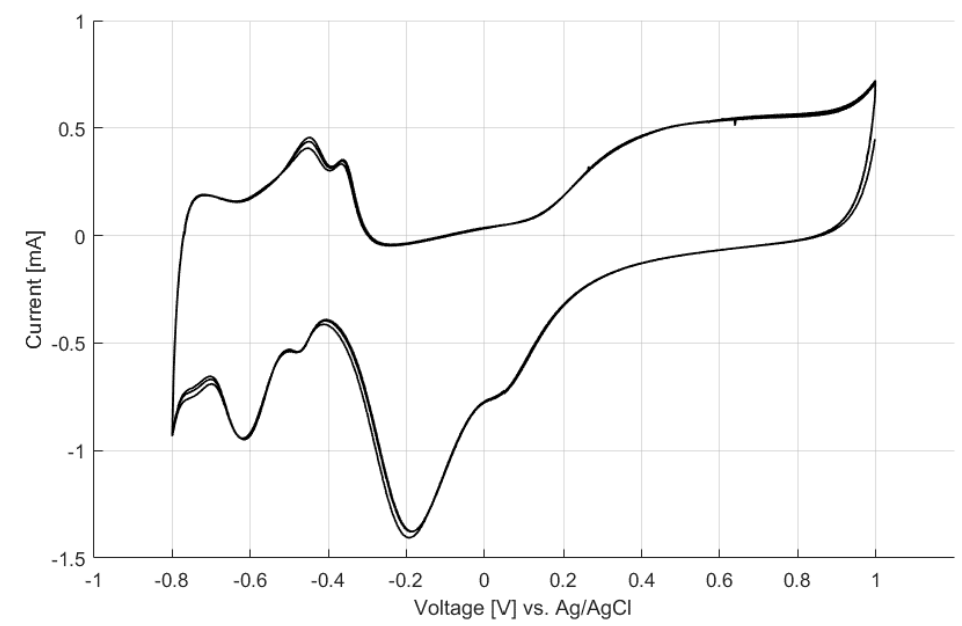

Fig. 6. Cyclic voltammogram of a single electrode of the fabricated array, showing hydrogen evolution around $-0.4 \mathrm{~V}$ and oxygen evolution around $1 \mathrm{~V}$.

\footnotetext{
${ }^{1}$ This averaging operation is necessary to limit the number of test parameters in order to avoid a too stringent multiple-comparison correction and to avoid dependencies between the samples when applying a statistical test.
} 


\subsection{Electrical impedance spectroscopy}

The impedance value of the electrode, submerged in PBS, was measured to be $2330 \pm 430 \Omega$ at 1 $\mathrm{kHz}$. This value is well below the input impedance of the amplifiers used during the in vivo experiment $(16 \mathrm{M} \Omega$ at $1 \mathrm{kHz})$ and are thus acceptable for physiological measurements. Impedance values which are too high would result in signal distortion and a low signal-to-noise ratio. [29]

\subsection{Controlled unfolding}

After introducing the electrode into the phantom in a similar manner as the in vivo implantation procedure described above, CT-scans were taken of the sample and reconstructed in 3D. A quarter section of the reconstructed image is shown in figure 7. The resulting image clearly shows that the electrode tip unfolded properly and that the flower electrode is radio-opaque enough. Moreover, it bends according to the dummy cavity shape and is in close contact with the dummy cavity wall.

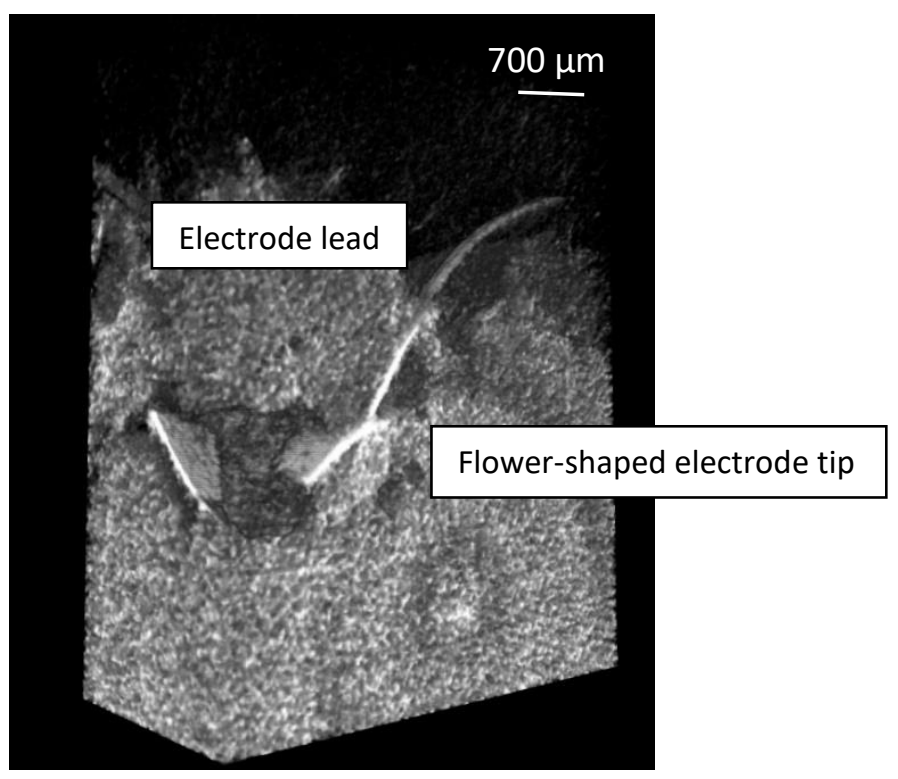

Fig. 7. CT-scan (quarter section) of the electrode after test implantation in a brain tissue phantom 


\subsubsection{CT- and MRI-imaging}

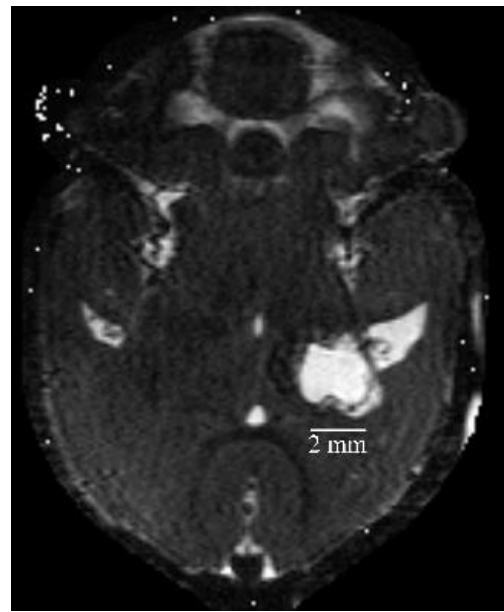

Fig. 8. MRI-scan showing the location and size of the created brain cavity (transversal cut through the anterior commissure, located $7 \mathrm{~mm}$ ventrally from bregma)

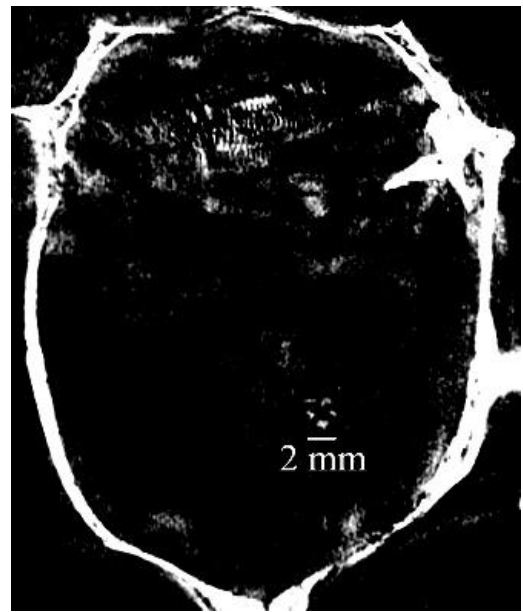

Fig. 9. CT-scan showing the electrode position (transversal cut through the anterior commissure, located $7 \mathrm{~mm}$ ventrally from bregma)

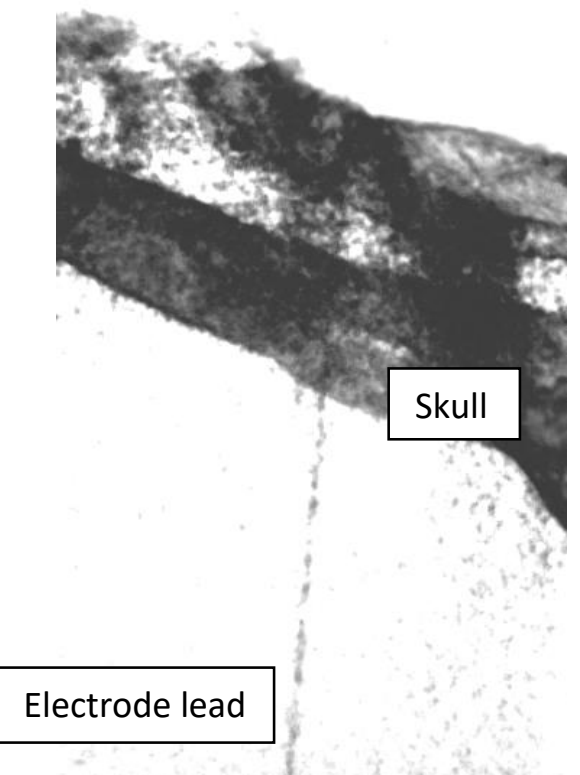

Flower-shaped electrode

The pre-implantation MRI-scan clearly showed the presence of the created cavity in the brain of the animal. An example of such a scan is shown in figure 8 . The diameter of the cavity is approximately 2 $\mathrm{mm}$. In the post-implantation CT-scan (fig. 9) the position of the electrode is visible. The scan shows the 7 individual electrode contacts which are unfolded within the cavity. An inter-electrode distance of $2 \mathrm{~mm}$ confirms that the electrode contacts have unfolded and are presumably in close proximity of the cavity wall. The post-implantation CT-scan shows the completely unfolded electrode, taking on a 3 dimensional shape conformal to the cavity

$1 \mathrm{~mm}$

Fig. 10. CT-scan (sagittal body plane) showing the unfolded electrode after implantation wall (fig. 10).

Based on the MR images, the aBCs of 21 rats were considered favorable for electrode implantation surgery (at least $1 \mathrm{aBC}$ with dimensions $>1 \times 1 \times 1.5 \mathrm{~mm}$ ). The remaining 3 rats were euthanized. Following post-implantation CT imaging and LFP recording, 11 additional rats were euthanized as the electrode in these rats appeared not to have unfolded and probably was still within the cannula. Although this appears to be a relatively low success ratio, it is still considerable given the complexity of the electrode preparation, stereotaxic calculations and surgical procedure. 


\subsubsection{Analysis LFP results}

Each of the LFP parameters (RMS voltage and relative band powers) was compared between movement and resting conditions using a Wilcoxon rank-sum test (also known as the MannWhitney U test), to detect significant differences between both conditions. Significance levels are set to $\alpha=0.05\left(^{*}\right)$ and Bonferroni correction was applied to account for multiple comparisons (6

parameters), resulting in a significance level of $\alpha=\frac{0.05}{6}=0.008(* *)$. The results of the LFP analysis are represented in the boxplots shown in figure 11 (comparing movement and resting states). The analyses were performed on an individual level (per rat) since the cavity size, lesion location and regions affected by the lesion differed for each rat. After a visual inspection of the LFP signals, 3 more rats were excluded due to faulty data.

A figure of the PSDs recorded in all seven electrodes implanted in one of the rats is illustrated in Fig. 12. The codes used to label the rats were for internal use and have no meaning in this context.

For 5 out of 7 rats, there is at least one of the six parameters that show a significance difference (**) between the movement and resting conditions, mostly in the RMS and the relative delta and theta band powers. In general, although clear differences can be observed between the movement and resting phases within each rat, no common trends were observed across the different rats, which is probably due to differences in the anatomical location of the electrodes and the aBC wall.

When comparing neural recordings between movement and resting phases, it is important to validate that the differences are indeed caused by changes in neural activity, and that they are not dominated by changes induced by motion artefacts. We have several reasons to believe that the observed differences are not artefact-related. First, it is noted that potential motion artefacts induced by cable movements are avoided due to the use of a wireless head stage. Furthermore, as can be seen from figure 11 it is observed that the relative delta band power is never higher during movement compared to resting (and in most cases even significantly lower), whereas most of the energy of motion artefacts is typically concentrated within this band. Furthermore, strong motionrelated artefacts would dominate over the actual LFP signals and would result in a very similar recorded signal in all 7 electrodes, which is clearly not the case from a visual comparison between PSDs corresponding to the different electrodes (figure 12). To test whether these differences are significant for each rat, we applied a Friedman test which compares the relative power in each subband across the 7 electrodes. Within each rat, significant differences were found across the electrodes for almost all of the 5 subbands, using a Bonferroni-corrected significance level $\alpha=$ 0.01 (the only exceptions were gammal in rat R1, alpha in rat R6, and delta in rat R5). 

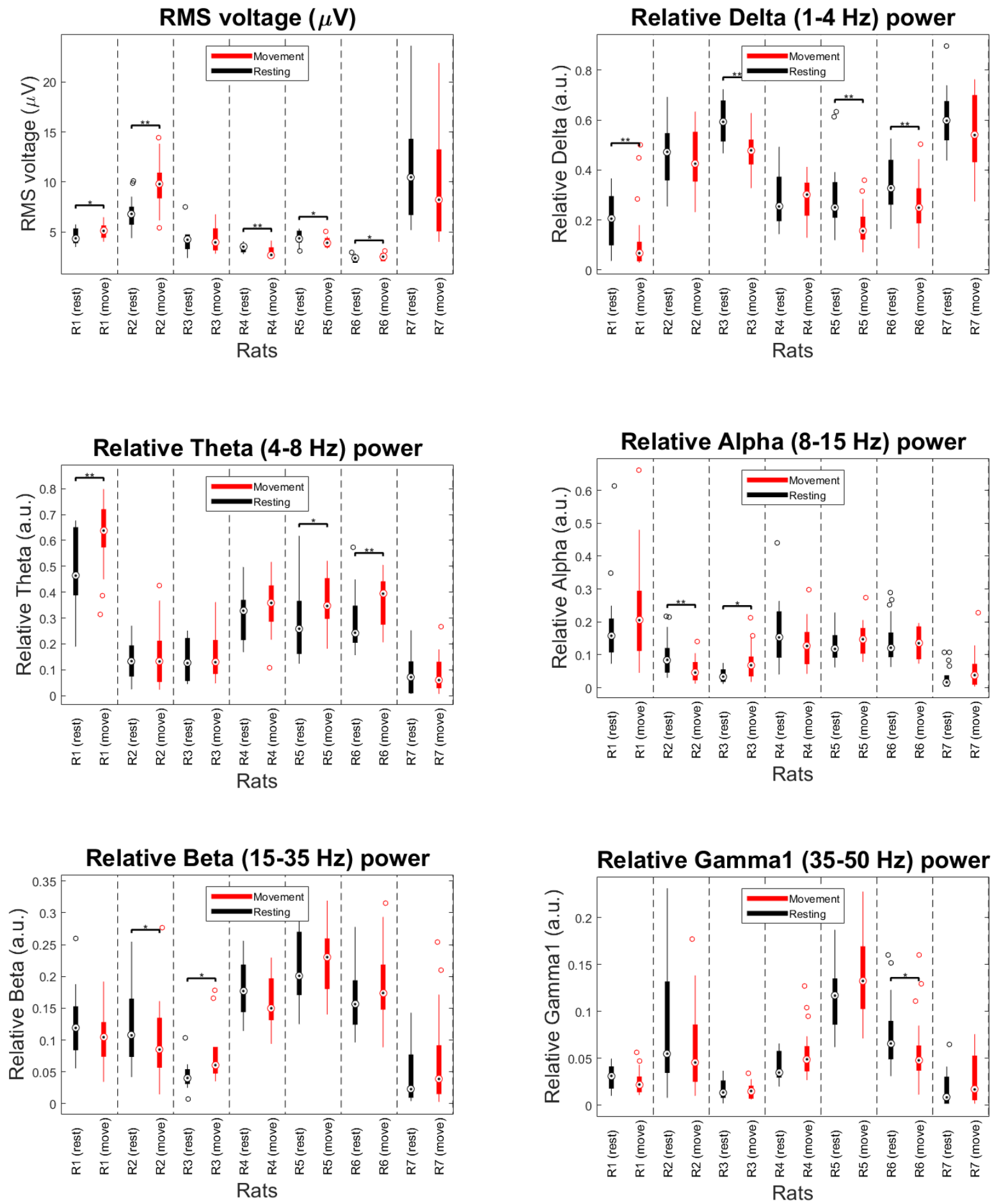

Fig. 11. Rat-level boxplots of the recorded LFP data depicting RMS voltage and relative power of different subbands during movement (red) and resting (black) phases. 
If we take a closer look at the PSD spectra of individual electrode contacts, we observe very clear differences between the PSDs in each electrode. Indeed, in Fig. 12, a large peak can be seen in the theta-band around $6 \mathrm{~Hz}$ on contacts 1, 5, 6 and 7 during movement. Other contacts show a smaller peak at around the same frequency, possibly due to a larger distance to the functional region in the brain from which the signal originates.
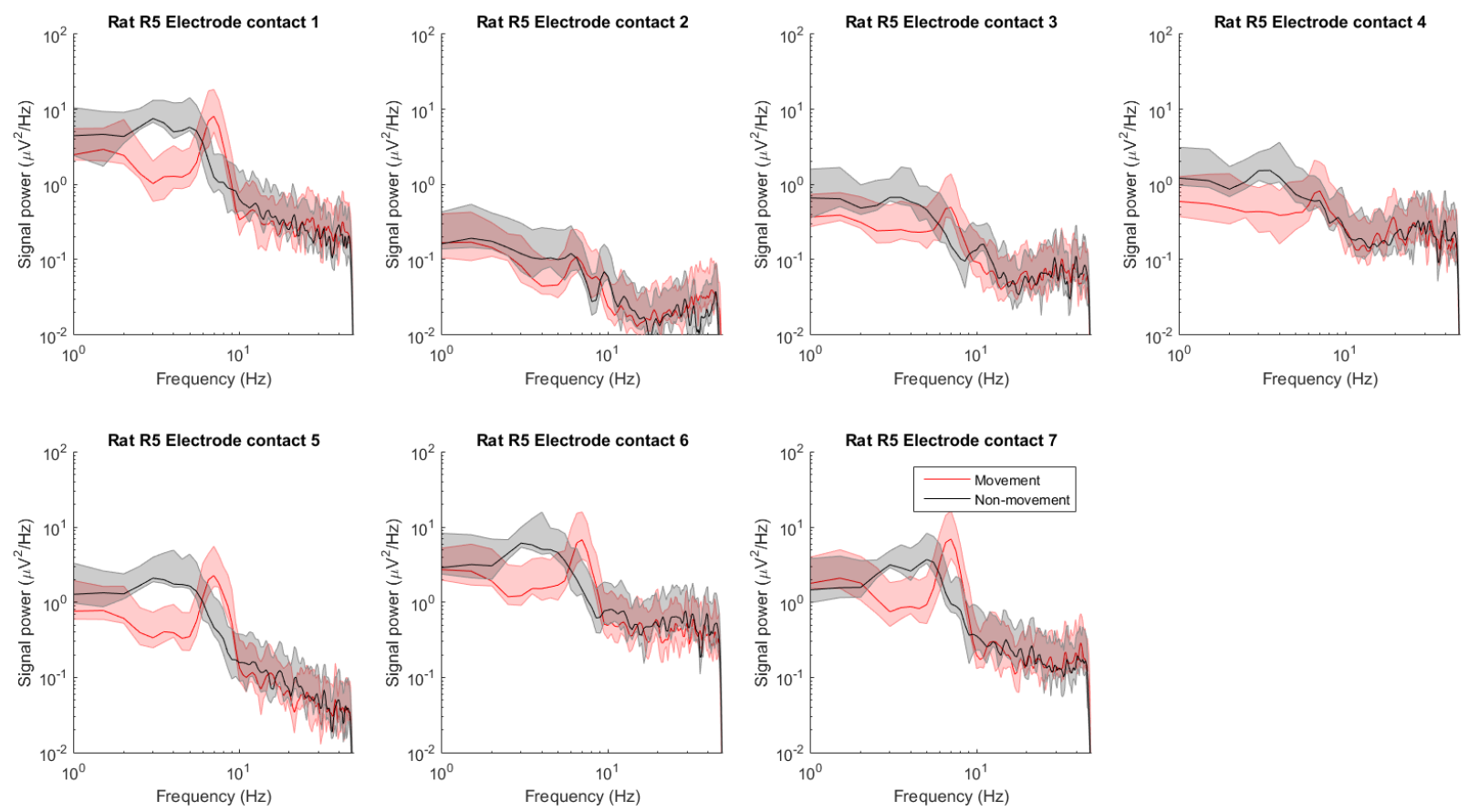

Fig. 12. PSD plot of the electrode contacts of rat $R 5$ in function of frequency. The shaded areas denote the $25 \%$ and $75 \%$ quartiles.

\section{Conclusion}

In summary, we have been able to design and fabricate a new thin-film neural electrode, specifically adapted for aBC electrical recording and stimulation. It was implanted correctly both ex vivo and in vivo, using a bespoke implantation procedure in which the electrode is temporarily folded onto itself and advanced through a cannula, minimizing damage to the healthy tissue surrounding the implantation trajectory. Although the main limitation of the device in its current design is the unfolding reliability, the results indicate that this technique has a lot of potential. Analysis of the recorded LFP data indicates that it is possible to record a signal which is not dominated by motion-related artefacts. Active neural elements were detected in the cavity wall which are possible targets for neuromodulation.

We believe that this design is a first proof of concept in the development of expandable neural probes which are custom made based on the geometry of the target cavity. We want to evolve to a pre-formed device which actively expands and covers the whole inner surface of the brain cavity wall. The use of foldable neural implants in research concerning aBC-associated diseases could open up a whole new target for neuromodulation. 


\section{Acknowledgements}

The research leading to these results has received funding from the European Research Council under the European Union's Seventh Framework Programme (FP7/2007-2013) / ERC grant agreement $n^{\circ} 340931$, and the KU Leuven IDO program, project on electrical brain stimulation IDO/12/024, and FWO Research Grants G0A5513N and G0D7516N, and the 2016 Research Grant of the European Society for Functional and Stereotactic Neurosurgery. 


\section{References}

[1] Sofroniew N J, Vlasov Y A, Hires S A, Freemand J and Svoboda K 2015 Neural coding in barrel cortex during whisker-guided locomotion eLIFE Sciences 4

[2] The National Collaborating Centre for Chronic Conditions 2006 Surgery for Parkinson's disease Parkinson's Disease pp 101-11

[3] Handa G 2006 Neural Prosthesis - Past, Present and Future Indian Journal of Physical Medicine \& Rehabilitation 171

[4] G. Schott 1996 From thalamic syndrome to central poststroke pain J. Neurol. Neurosurg. Psychiatry 61 560-564

[5] Kalita $\mathbf{J}$ and Vajpayee $\mathbf{J} 2006$ Comparison of rednisolone with piroxicam in complex regional pain syndrome following stroke: a randomized controlled trial QJM 99 89-95

[6] Sutton A C et al 2013 Deep brain stimulation of the substantia nigra pars reticulata improves forelimb akinesia in the hemiparkinsonian rat J. Neurophysiol. 109 363-374

[7] Kleim J A et al 2003 Motor cortex stimulation enhances motor recovery and reduces periinfarct dysfunction following ischemic insult Neurol. Res. 25 789-793

[8] Teskey G C, Flynn C, Goertzen C D, Monfils M H and Young N A 2003 Cortical stimulation improves skilled forelimb use following a focal ischemic infarct in the rat Neurol. Res. 25 794800

[9] Gulati T et al 2015 Robust neuroprosthetics control from the stroke perilesional cortex $J$. Neurosci. Off. J. Soc. Neurosci. 35 8653-8661

[10] Yamgoue Y, Pralong E, Levivier M and Bloch J 2016 Deep Brain Stimulation of the Ventroposteromedial (VPM) Thalamus 10 Years after VPM Thalamotomy to Treat a Recurrent Facial Pain Stereotact. Funct. Neurosurg. 94 118-122

[11] Waln O and Jankovic J 2013 Bilateral globus pallidus internus deep brain stimulation after bilateral pallidotomy in a patient with generalized early-onset primary dystonia Mov. Disord. Off. J. Mov. Disord. Soc. 28 1162-1163

[12] Butler J T, Rodriguez W, Pallavaram S, Konrad P and Tolleson C M 2016 Thalamic deep brain stimulation as rescue therapy for tremor following bilateral radiosurgery Parkinsonism Relat. Disord. 36 100-102

[13] Liu J et al 2015 Syringe injectable electronics. Nature Nanotechnology. 10 629-636

[14] Hure J, Roman B and Bico J 2011 Wrapping an adhesive sphere with an elastic sheet Physical review letters 106174301

[15] Rubehn B and Stieglitz T 2010 In vitro evaluation of the long-term stability of polyimide as a material for neural implants Biomaterials 31 3449-3458 
[16] Stieglitz T, Beutel H, Schuettler M, Meyer J U 2000 Micromachined, polyimide-based devices for flexible neural interfaces Biomedical Microdevices 2 283-294

[17] Ahmed Jarboui et al 2013 Parylene-C for hermetic and flexible encapsulation of interconnects and electronic components 4th Flexible and Stretchable Electronics conference, Abstracts

[18] Meng E 2010 biomedical microsystems CRC Press

[19] Ceyssens F, van Kuyck K, Vande Velde G, Welkenhuysen M, Stappers L, Nuttin B and Puers B 2013 Resorbable scaffold based chronic neural electrode arrays Biomedical Microdevices 15(3) 481-493

[20] Ceyssens F and Puers B 2015 Insulation lifetime improvement of polyimide thin film neural implants Journal of Neural Engineering 12(5) 054001

[21] Brummer S B and Turner M J 1975 Electrical stimulation of the nervous system: The principle of safe charge injection with noble metal electrodes Bioelectrochemistry and Bioenergetics 2 1325

[22] Brummer S B and Turner M J 1977 Electrochemical considerations for safe electrical stimulation of the nervous system with platinum electrodes IEEE Transactions on Biomedical Engineering 24 59-63

[23] Brummer S B and Turner M J 1977 Electrical Stimulation with Pt Electrodes: A Method for Determination of" Real” Electrode Areas IEEE Transactions on Biomedical Engineering 24 436439

[24] Brummer S B and Turner M J 1977 Electrical Stimulation with Pt Electrodes: II Estimation of Maximum Surface Redox (Theoretical Non-Gassing) Limits IEEE Transactions on Biomedical Engineering 24 440-443

[25] Cooperrider J, Gale J T, Gopalakrishnan R, Chan H H, Wathen C, Park H J, Baker K B, Shaikh A G and Machadon A G 2016 Differential frequency modulation of neural activity in the lateral cerebellar nucleus in failed and successful grasps Experimental Neurology 277 27-34

[26] Hudak E M, Mortimer J T and Martin H B 2010 Platinum for neural stimulation: voltammetry considerations J. Neural Eng. 726005

[27] Musa S, Welkenhuysen M, Prodanov D, Eberle W, Bartic C, Nuttin B and Borghs G 2009 In vitro and in vivo electrochemical characterization of a microfabricated neural probe Conf Proc IEEE Eng Med Biol Soc 7143-6

[28] Musa S 2011 Design Fabrication and Characterization of a Neural Probe for Deep Brain Stimulation and recording (Doctoral dissertation)

[29] Fontes M B A 2013 Electrodes for bio-application: recording and stimulation J. Phys. Conf. Ser. 421 